\title{
La Unión Europea y su problemática de integración
}

The European Union and its integration problem

\section{Santiaga Ánima Puentes *}

* Profesora Titular de tiempo completo en la Facultad de Economía, UNAM. Coordinadora del Centro de Estudios Económicos de la Unión Europea-México (CEEUEM).
Palabras clave

Economía internacional, Integración económica,

Politica comercial,

Key words

International Economics, Economic Integration,

Trade Negotiations

JEL

F, F02, F13

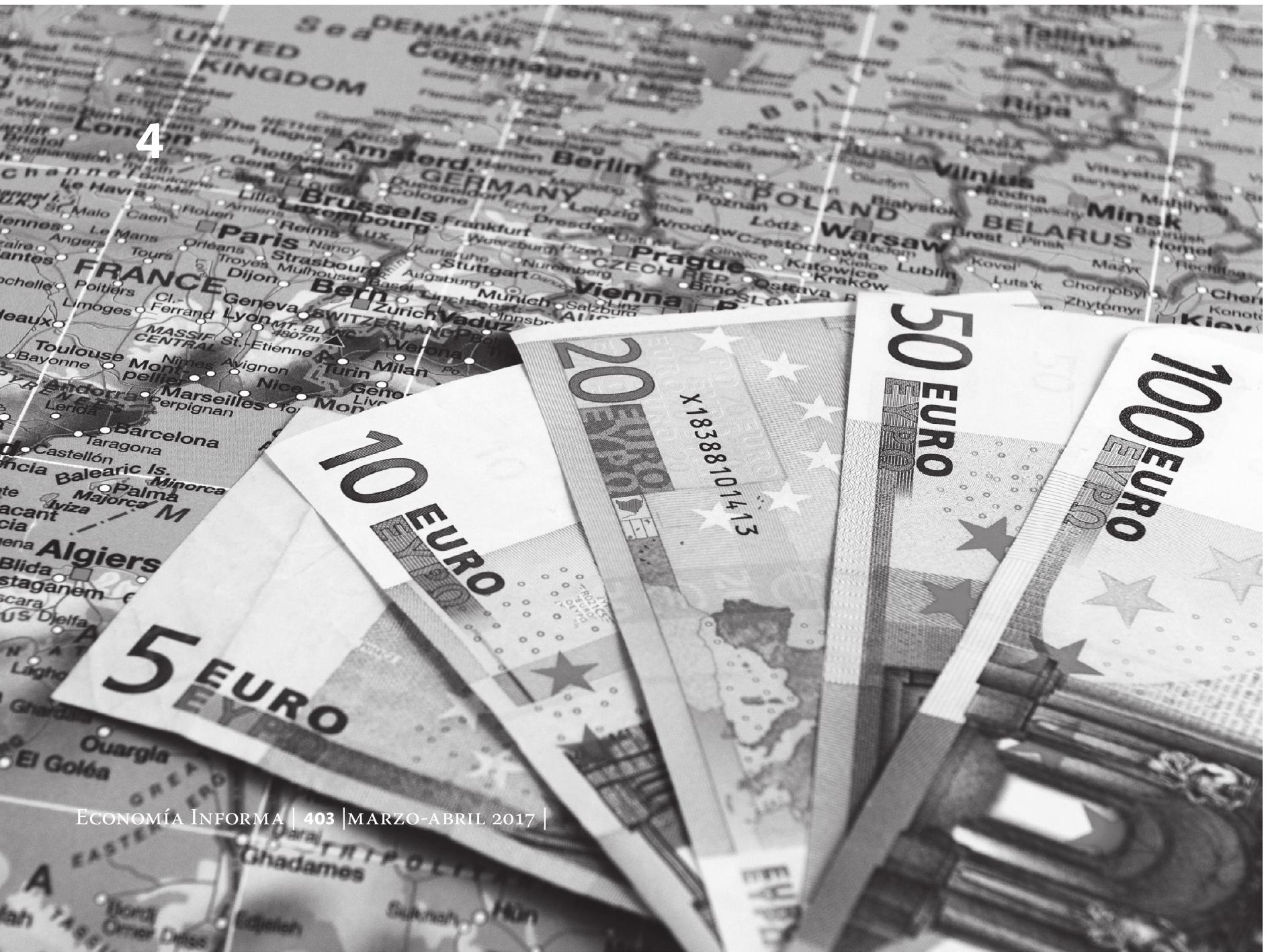




\section{Santiaga Ánima Puentes | La Unión Europea y su problemática de integración}

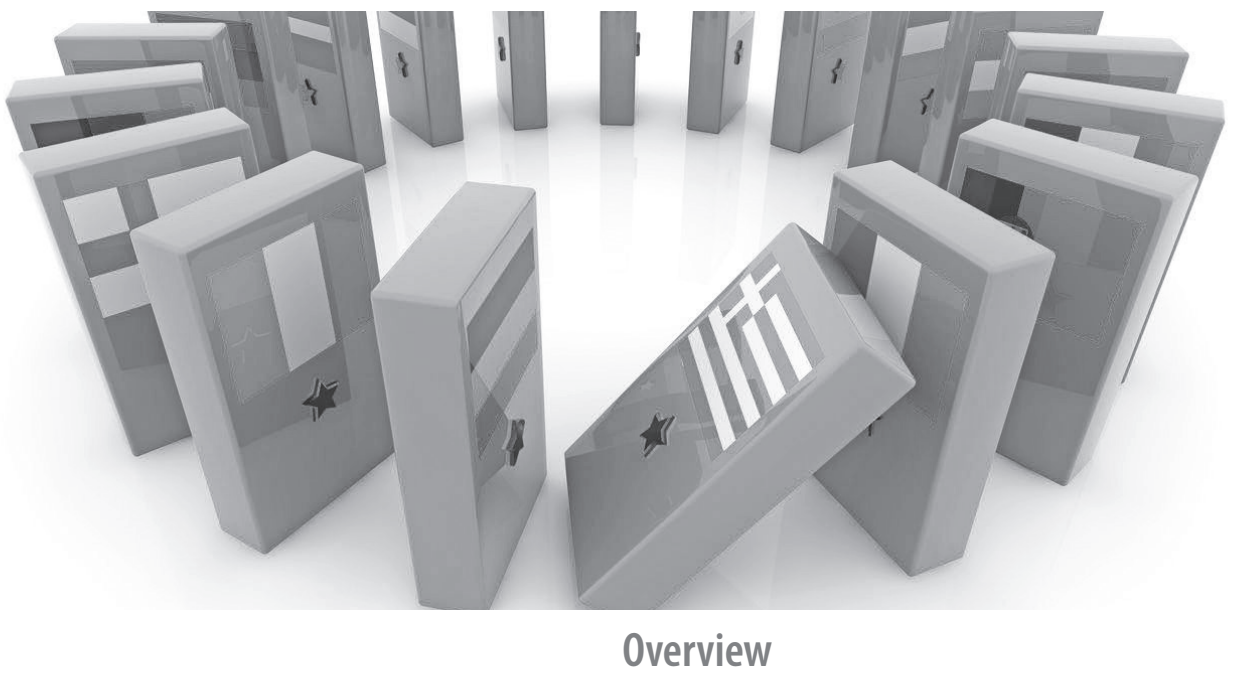

\section{Resumen}

La Unión Europea ha enfrentado en los últimos años serios problemas que han puesto en duda su viabilidad, frente a esa situación se plantea en este trabajo cuáles serían las posibles vías por las cuales los países integrantes de la Unión pueden superar las dificultades de la integración. Europa se ha visto cimbrada por la crisis global y ha mostrado su vulnerabilidad frente a embates externos de carácter financiero; en ese sentido, la Unión Europea no se ha visto fortalecida como era el objetivo de su creación.

Los indicadores económicos han mostrado que los países de la Unión Europea están enfrentando fluctuaciones en su actividad económica a partir de la recesión de 2009, obligando a responder en un primer momento con políticas expansivas de la demanda agregada vía aumento del déficit público y de los niveles de endeudamiento, y en un segundo momento con políticas de ajuste y reducción del gasto.

El contexto de crisis global en el cual la Unión Europea es un protagonista central la obliga a tener una mayor participación económica y social. El mayor reto que tiene es resolver sus problemas internos, una vez que lo logre podrá actuar en bloque frente a problemas provenientes de fuera de la zona europea, sino lo resuelven, se acrecentaran los conflicto económicos y sociales, mismos que pueden complicar más el escenario actual y retardar el proceso de integración europea.
The European Union has faced in recent years serious problems that have called into question its viability, considering these facts, this work shares possible routes by which members of the Union can overcome the difficulties of the integration process. Europe has been shaken by the global crisis and has shown its vulnerability to external financial shocks; in this regard, the European Union has not been strengthened as it was intended with its creation.

The economic indicators have shown that the countries of the European Union are facing constant fluctuations in their economic activity since the recession of 2009, forcing to respond initially, with expansionary policies in aggregate demand through increased public deficit and rising debt levels, and secondly, with spending reduction and adjustment policies.

The context of the global crisis in which the European Union is a central player, requires it to have a greater economic and social participation. Solving their internal problems is the Union's biggest challenge and once achieved, it will stand in a better position to act in unity facing problems from outside the euro zone, However, if these internal problems are not solved, the result will be an increase in economic and social issues which could complicate even further the current scenario and at the same time, slow down the process of European integration. 


\section{Introducción}

El artículo se basa en las conclusiones obtenidas en el Seminario Semestral organizado por Centro de Estudios Económicos de la Unión Europea-México (CEEUEM), sobre la situación económico-social de la Unión Europea (UE); por lo mismo, incorpora algunas discusiones estructurales y los efectos de las coyunturas existentes en esta región.

La Unión Europea ha enfrentado en los últimos años serios problemas que han puesto en duda su viabilidad de integración, en consecuencia, nos enfrentamos a un escenario donde surgen algunos interrogantes de la viabilidad del proceso de integración.

Cuáles son las principales dificultades que enfrenta el proceso de integración en su actual fase y cuáles pueden ser las formas de superación de las mismas.

La Unión Europea se vio sacudida por la crisis que afrontaron varios de los países integrantes, entre los más destacados se encuentran algunos de los países de reciente integración a la Unión, como Chipre, además de países que ya venían formando parte de Unión como Grecia, España, Portugal e Italia. Para el caso particular de Ucrania el contexto de la problemática de su posible incorporación a la Unión, tuvo tintes políticos y estratégicos y no solo a cuestiones de integración económica.

El horizonte temporal del trabajo abarca el periodo que va 2007 a 2014 y su exposición se presenta en cuatro apartados, en los que se destaca como los niveles de la demanda agregada, han afectado la actividad económica y mostrando la fragilidad de la integración económica Europea frente a la inestabilidad de los mercados financieros internacionales, así en el primer apartado se examina las causas recientes de la crisis y sus efectos en la integración; el segundo aborda uno de los rasgos más notorios de dicha crisis que son los altos niveles de endeudamiento de algunos de sus países miembros de la UE (Grecia, España entre otros) que violentan las disposiciones del Tratado de Maastricht, en el tercer apartado se muestra como se han presentado los aumentos en el déficit público a raíz de la crisis financiera y económica mundial; y el cuarto revisa como mediante una política keynesiana, de expansión del gasto público, los países integrantes de la Unión Europea reaccionaron ante los efectos devastadores en el empleo y caída en la demanda agregada y que como hemos seńalado fueron ocasionados por la crisis financiera global proveniente del mercado financiero norteamericano. Por último, el artículo concluye que la reciente inestabilidad económica global y financiera mostró la vulnerabilidad del proceso de integración económica de la UE y que frente a la magnitud de la crisis, las respuestas de una mayor intervención del estado en la economía, a través de expansión del gasto público, para revitalizar la demanda agregada apenas fue una respuesta limitada que no ha resuelto como avanzar en la integración de aquellos países que presentan problemas de insuficiente capital de inversión para crecer, y que su situación se agudiza por los efectos globalizadores de la crisis.

\section{Crisis Europea}

Las causas recientes de la crisis enfrentada desde hace varios años por la Unión Europea tienen su origen en la crisis de 2008. Esta crisis se generó por la situación de insolvencia de los deudores de créditos hipotecarios, lo que afecto el valor de los instrumentos financieros a la baja ocasionando la caída de instituciones financieras como Lehman Brothers, en los Estados Unidos y su posterior transmisión hacia Europa. 


\section{Santiaga Ánima Puentes | La Unión Europea y su problemática de integración}

Estas causas se encuentran en los altos niveles de endeudamiento que tenían las instituciones financieras europeas, quienes de inmediato resintieron la ola de la crisis.

En el Reino Unido el sistema financiero se vio seriamente comprometido obligando al Banco de Inglaterra a intervenir para rescatar a instituciones financieras al borde de la quiebra. En el caso de los demás integrantes de la UE, los efectos se dejaron sentir no solo en el sistema financiero sino que también repercutieron en las actividades productivas; la crisis de $2009^{1}$ afectó seriamente el PIB de los países de la Unión como puede verse en las tasas de crecimiento de la gráfica 1 . y que afectaron el sector real de la actividad productiva, inhibiendo los niveles de inversión y contrayendo la demanda agregada, tales fueron los casos de Italia, Suecia, Dinamarca, Luxemburgo, Grecia, Irlanda, Estonia, Letonia y Reino Unido. Las causas de la caída en la actividad económica se derivan de los efectos que el sector financiero de Estados Unidos trasmitió hacia el resto de las economías, adicionalmente la falta de control en el mercado financiero que permitiera regular los flujos de inversión financiera acentuaron los niveles de especulación prevalecientes en los mercados mundiales de capitales, lo que ocasionó que

\section{Gráfica 1 Tasas de crecimiento UE(28), Alemania, Francia e Italia 2003-2014}

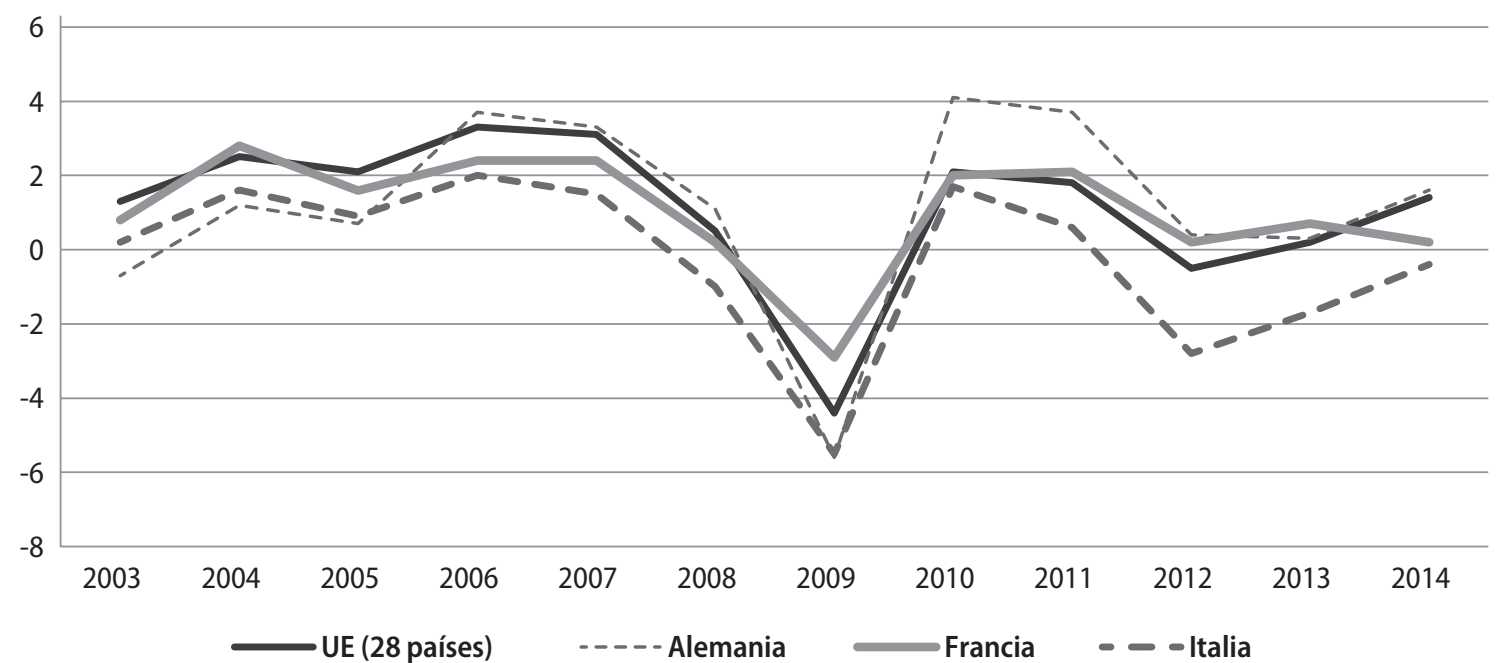

Fuente: elaboración propia con datos de la página de Eurostat.

Las tasas de crecimiento de los 28 países que integran la Unión registran una caída generalizada en sus tasas de crecimiento en el año de 2009 (Cuadro 1), aunque algunos países de la UE empezaron a resentir los efectos desde 2008, debido a la crisis financiera proveniente de los mercados financieros de Estados Unidos

1 Para la ue los efectos de la crisis se manifestaron a partir del 2009 teniendo su mayor efecto con la crisis griega en mayo del 2010. afectará las decisiones de inversión y consumo en los países de la Unión Europea.

Para los habitantes de Alemania asumir los costos de la integración ha sido una crítica constante

“...ंpero por qué es necesario que paguen los trabajadores alemanes en vez de los griegos acaudalados?..."

2 Jean Pisani-Ferry. (2012). El despertar de los demonios. Editorial Antoni Bosch, p. 13. 
Ante el descontento de los ciudadanos alemanes que observan como su gobierno sostiene a países que no aportan al desarrollo de la Unión y si en cambio, según aprecian, se ven aparentemente beneficiados con recursos provenientes del país que prácticamente sostienen a la Unión, como es el caso de Alemania, lo anterior indica que el proceso de integración plantea un dilema continuar con los actuales países o dejar que los países más vulnerables dejen la Unión y no dificulten la integración de la misma.

Cuadro 1

Producto Interno Bruto Unión Europea 2003-2014. Tasas de crecimiento anual

\begin{tabular}{|c|c|c|c|c|c|c|c|c|c|c|c|c|}
\hline País/Año & 2003 & 2004 & 2005 & 2006 & 2007 & 2008 & 2009 & 2010 & 2011 & 2012 & 2013 & 2014 \\
\hline UE (28 países) & 1.3 & 2.5 & 2.1 & 3.3 & 3.1 & 0.5 & -4.4 & 2.1 & 1.8 & -0.5 & 0.2 & 1.4 \\
\hline Bélgica & 0.8 & 3.6 & 2.1 & 2.5 & 3.4 & 0.7 & -2.3 & 2.7 & 1.8 & 0.2 & 0 & 1.3 \\
\hline Luxemburgo & 1.4 & 4.4 & 3.2 & 5.1 & 8.4 & -0.8 & -5.4 & 5.7 & 2.6 & -0.8 & 4.3 & 4.1 \\
\hline Países Bajos & 0.3 & 2 & 2.2 & 3.5 & 3.7 & 1.7 & -3.8 & 1.4 & 1.7 & -1.1 & -0.5 & 1 \\
\hline Alemania & -0.7 & 1.2 & 0.7 & 3.7 & 3.3 & 1.1 & -5.6 & 4.1 & 3.7 & 0.4 & 0.3 & 1.6 \\
\hline Francia & 0.8 & 2.8 & 1.6 & 2.4 & 2.4 & 0.2 & -2.9 & 2 & 2.1 & 0.2 & 0.7 & 0.2 \\
\hline Italia & 0.2 & 1.6 & 0.9 & 2 & 1.5 & -1 & -5.5 & 1.7 & 0.6 & -2.8 & -1.7 & -0.4 \\
\hline Reino Unido & 3.3 & 2.5 & 3 & 2.7 & 2.6 & -0.5 & -4.2 & 1.5 & 2 & 1.2 & 2.2 & 2.9 \\
\hline Irlanda & 3.8 & 4.4 & 6.3 & 6.3 & 5.5 & -2.2 & -5.6 & 0.4 & 2.6 & 0.2 & 1.4 & 5.2 \\
\hline Dinamarca & 0.4 & 2.6 & 2.4 & 3.8 & 0.8 & -0.7 & -5.1 & 1.6 & 1.2 & -0.1 & -0.2 & 1.3 \\
\hline Grecia & 5.8 & 5.1 & 0.6 & 5.7 & 3.3 & -0.3 & -4.3 & -5.5 & -9.1 & -7.3 & -3.2 & 0.7 \\
\hline España & 3.2 & 3.2 & 3.7 & 4.2 & 3.8 & 1.1 & -3.6 & 0 & -1 & -2.6 & -1.7 & 1.4 \\
\hline Portugal & -0.9 & 1.8 & 0.8 & 1.6 & 2.5 & 0.2 & -3 & 1.9 & -1.8 & -4 & -1.1 & 0.9 \\
\hline Austria & 0.8 & 2.7 & 2.1 & 3.4 & 3.6 & 1.5 & -3.8 & 1.9 & 2.8 & 0.8 & 0.3 & 0.4 \\
\hline Finlandia & 2 & 3.9 & 2.8 & 4.1 & 5.2 & 0.7 & -8.3 & 3 & 2.6 & -1.4 & -1.1 & -0.4 \\
\hline Suecia & 2.4 & 4.3 & 2.8 & 4.7 & 3.4 & -0.6 & -5.2 & 6 & 2.7 & -0.3 & 1.2 & 2.3 \\
\hline Polonia & 3.6 & 5.1 & 3.5 & 6.2 & 7.2 & 3.9 & 2.6 & 3.7 & 5 & 1.6 & 1.3 & 3.3 \\
\hline República Checa & 3.6 & 4.9 & 6.4 & 6.9 & 5.5 & 2.7 & -4.8 & 2.3 & 2 & -0.9 & -0.5 & 2 \\
\hline Hungría & 3.8 & 4.9 & 4.4 & 3.8 & 0.4 & 0.8 & -6.6 & 0.7 & 1.8 & -1.7 & 1.9 & 3.7 \\
\hline Eslovenia & 2.8 & 4.4 & 4 & 5.7 & 6.9 & 3.3 & -7.8 & 1.2 & 0.6 & -2.7 & -1.1 & 3 \\
\hline Eslovaquia & 5.4 & 5.3 & 6.4 & 8.5 & 10.8 & 5.7 & -5.5 & 5.1 & 2.8 & 1.5 & 1.4 & 2.5 \\
\hline Estonia & 7.4 & 6.3 & 9.4 & 10.3 & 7.7 & -5.4 & -14.7 & 2.5 & 7.6 & 5.2 & 1.6 & 2.9 \\
\hline Letonia & 8.4 & 8.3 & 10.7 & 11.9 & 10 & -3.6 & -14.3 & -3.8 & 6.2 & 4 & 3 & 2.4 \\
\hline Lituana & 10.5 & 6.6 & 7.7 & 7.4 & 11.1 & 2.6 & -14.8 & 1.6 & 6 & 3.8 & 3.5 & 3 \\
\hline Malta & 2.5 & 0.4 & 3.8 & 1.8 & 4 & 3.3 & -2.5 & 3.5 & 2 & 2.9 & 4 & 4.1 \\
\hline Chipre & 2.8 & 4.6 & 3.9 & 4.5 & 4.9 & 3.7 & -2 & 1.4 & 0.4 & -2.4 & -5.9 & -2.5 \\
\hline Bulgaria & 5.1 & 6.6 & 7.2 & 6.8 & 7.7 & 5.6 & -4.2 & 0.1 & 1.6 & 0.2 & 1.3 & 1.5 \\
\hline Rumania & 4.8 & 6.8 & 4.3 & 6.8 & 7.1 & 6.8 & -5.6 & -1 & 1.1 & 2.4 & 3 & 2.9 \\
\hline Croacia & 5.6 & 4.1 & 4.2 & 4.8 & 5.2 & 2.1 & -7.4 & -1.7 & -0.3 & -2.2 & -0.9 & -0.4 \\
\hline
\end{tabular}

Fuente: elaborado con datos del sitio web de la Unión Europea.

http://ec.europa.eu/eurostat/tgm/table.do?tab=table\&init=1\&language=en\&pcode=tec00115 
La parte del ciclo económico recesivo fue el resultado de la crisis financiera mundial y que especialmente algunos ${ }^{3}$ de los 18 países que integran la zona euro resintieron la crisis generalizada de 2009, su situación crítica se reflejó en la agudización de los problemas de endeudamiento público y gasto gubernamental, ambos superiores a su capacidad de endeudamiento y a sus ingresos públicos, ocasionando un gigantesco aumento del déficit público, a pesar de los requisitos tan restrictivos que se asentaron en el Tratado de Maastricht. ${ }^{4}$

El promedio de crecimiento económico de los 28 países de la UE tuvo un comportamiento fluctuante entre 2003-2007, que se agudizo en 2008 y 2009 con la crisis global, hubo después un ligero repunte en 2010, sin embargo en los años de 2011 a 2014 el promedio de crecimiento ha sido errático sin tener un ritmo sostenido en el aumento de la actividad económica. Como ejemplo Alemania, la principal economía de la UE, a pesar de que se recuperó en 2010, tuvo un decrecimiento de 2011 a 2013 y para 2014 apenas obtuvo un ligero crecimiento de $1.6 \%$. Todo ello indica que la demanda agregada, en los países de la Unión Europea ha estado sujeta en el sector financiero al mercado global y a las recesivas fluctuaciones cíclicas de corto plazo en la actividad productiva, la respuesta ante esa situación fue una mayor intervención del Estado en la economía.

El restar soberanía monetaria a raíz de la creación de la zona euro y adaptarse al mer-

3 En particular Grecia aumento su nivel de endeudamiento hasta el $178.8 \%$, Chipre al 160 y Portugal en 133 en el primer trimestre de 2014 (cuadro 2) y el gasto gubernamental en 2013 Grecia lo aumentó hasta el $58.5 \% / \mathrm{PIB}$.

4 Fue firmado en 1992 y puesto en vigor en noviembre de 1993. En él se establecieron los denominados Criterios de Convergencia que impusieron el tope del $60 \% /$ PIB en la deuda y del $3 \%$ para el déficit, entre otros.

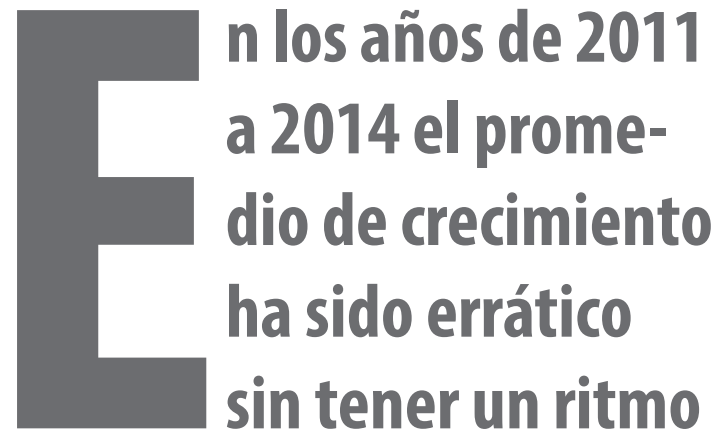

\section{sostenido en el aumento de la actividad económica}

cado monetario basado en una moneda única entre sus integrantes, limitó la capacidad de acción de los hacedores de política monetaria en cada uno de los países miembros, al subordinar los instrumentos de la política económica y cambiaria al sostenimiento de una tasa de interés y tipo de cambio.

Frente a esta situación los países integrantes de la Unión Europea se sujetaron al control de la política monetaria establecida por el Banco Central Europeo, ${ }^{5}$ dejando en cambio que los países tuvieran margen de acción en la política fiscal. Sin embargo la misma política fiscal tiene que equilibrar los desajustes monetarios a través de la reducción del déficit público y del nivel de endeudamiento, lo que sólo es posible si se reduce el gasto público, se aumentan los impuestos y se reestructura la deuda solicitando más créditos para cubrir el desbalance en los recursos públicos. ${ }^{6}$

Nuevamente recurrimos al ejemplo griego donde los distintos gobiernos de cualquier orientación política o ideológica tuvieron que

5 Como demostró la realidad no todos los países se sujetaron del todo, algunos maquillaron sus cifras, siendo Grecia el ejemplo más evidente.

6 Para una revisión del análisis de los desequilibrios macroeconómicos se encuentra en Girón y Solorza (2013) y Pisani-Ferry (2012). 
imponer medidas restrictivas que permitieron cumplir con los pagos mínimos de la deuda afectando el nivel de producto, los niveles salariales y de empleo. Además de producir la caída de las pensiones, el incremento de la edad de jubilación, entre otros efectos negativos para la población.

\section{Creciente endeudamiento}

El proceso de integración de la zona euro ha estado a prueba ${ }^{7}$ por los efectos negativos que se han dejado sentir sobre las economías europeas a partir de la crisis de 2009, el problema macroeconómico fundamental es el llamado problema de la deuda soberana que enfrentaron cinco ${ }^{8}$ de sus países miembros, este problema consiste en una deuda de magnitud superior al 100 por ciento de su PIB.

Los países que registraron esos montos elevados de endeudamiento del sector gubernamental, como se observa en el cuadro 2, con datos trimestrales de 2011 hasta el primero de 2014 son Grecia, España, Portugal, Italia, Irlanda y Chipre.

En el mismo cuadro, puede comprobarse que el promedio de la deuda pública de los 28 países que actualmente integran la UE, aumentó en el mismo periodo del 71.6 al 78.4 por ciento del pib, lo cual muestra el efecto de la crisis financiera mundial.

Como ya habíamos señalado estos datos indican una clara violación de lo establecido en el Tratado de Maastricht de mantener en un 60 por ciento el nivel de endeudamiento con respecto al PIB.

7 Es notorio por los cuestionamientos institucionales como el FMl, y por una variedad de opiniones desde los organismos internacionales hasta la opinión pública de los diferentes Países.

8 Los países son Italia, Grecia, Irlanda, Portugal y Chipre.

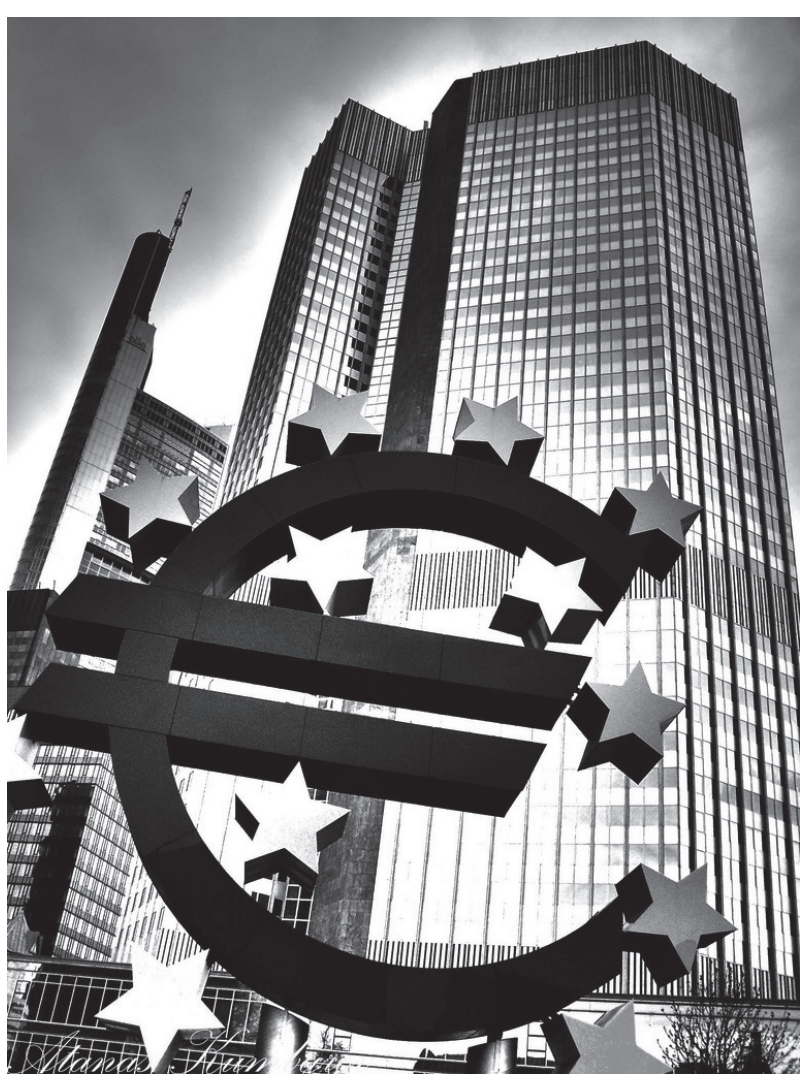

En el caso de España los efectos de la crisis se vieron reflejados en la creciente demanda de trabajo de la población joven y preparada, que se enfrentaban a un mundo laboral cada vez más restringido, y que con la recesión del ciclo de corto disparo los niveles de endeudamiento partir del 2012, que como ya se señaló superaron lo exigido por el Tratado de Maastricht; alcanzando el 84.5 por ciento de endeudamiento del PIB para el primer trimestre de 2014.

Lo anterior lo antecedió un aumento constante del ritmo de crecimiento económico, el cual se indica en el cuadro 1, observamos como la situación de España y Grecia en los años de 2003 a 2007 fue de un crecimiento económico positivo, sin embargo, este ritmo de crecimiento mostró su fragilidad frente a la crisis financiera global; en el caso de Portugal mostró, para los mismos años, un crecimiento económico precario de su actividad económica. 
Santiaga Ánima Puentes | La Unión Europea y su problemática de integración

Cuadro 2

Deuda Pública como porcentaje del PIB del gobierno central por países. Trimestral

\begin{tabular}{|c|c|c|c|c|c|c|c|c|c|c|}
\hline País/Año & 201104 & $2012 Q 1$ & 202102 & $2012 Q 3$ & $2012 Q 4$ & 201301 & $2013 Q 2$ & 201303 & 201304 & $2014 Q 1$ \\
\hline UE (28 países) & 71.6 & 72.4 & 74.2 & 74.5 & 75.1 & 76.0 & 77.2 & 77.4 & 77.6 & 78.4 \\
\hline Bélgica & 90.0 & 92.6 & 93.1 & 92.1 & 91.3 & 94.7 & 94.8 & 93.9 & 91.8 & 94.6 \\
\hline Luxemburgo & 17.0 & 19.6 & 19.9 & 19.9 & 20.0 & 21.5 & 21.3 & 26.2 & 21.5 & 21.3 \\
\hline Holanda & 58.9 & 60.3 & 62.1 & 63.5 & 64.6 & 65.4 & 67.2 & 67.1 & 67.1 & nd \\
\hline Alemania & 50.7 & 51.2 & 52.0 & 51.1 & 51.3 & 51.3 & 51.2 & 50.2 & 50.1 & 49.5 \\
\hline Francia & 71.2 & 72.9 & 75.4 & 74.6 & 75.8 & 77.3 & 79.1 & 78.5 & 78.6 & 80.1 \\
\hline Italia & 113.8 & 116.7 & 118.8 & 120.1 & 120.2 & 123.4 & 126.4 & 126.1 & 126.3 & 129.4 \\
\hline Reino Unido & 83.3 & 84.1 & 84.4 & 86.2 & 88.0 & 87.5 & 89.0 & 88.6 & 89.5 & 90.1 \\
\hline Irlanda & 103.6 & 106.3 & 109.7 & 116.7 & 117.0 & 124.5 & 125.0 & 124.0 & 123.3 & nd \\
\hline Dinamarca & 39.3 & 37.8 & 39.2 & 40.2 & 38.4 & 37.4 & 38.5 & 39.2 & 37.3 & 37.4 \\
\hline Grecia & 181.2 & 141.3 & 153.1 & 156.2 & 161.1 & 164.5 & 173.5 & 176.6 & 179.4 & 178.8 \\
\hline España & 59.5 & 62.8 & 65.5 & 67.2 & 73.9 & 77.7 & 80.0 & 81.3 & 81.7 & 84.5 \\
\hline Portugal & 106.3 & 109.9 & 116.3 & 118.9 & 123.7 & 127.1 & 131.0 & 129.0 & 129.1 & 133.0 \\
\hline Austria & 66.3 & 67.3 & 69.4 & 68.2 & 68.0 & 69.3 & 69.4 & 71.5 & 68.5 & 69.1 \\
\hline Suecia & 32.5 & 31.6 & 31.4 & 31.5 & 31.6 & 34.3 & 34.1 & 34.3 & 34.1 & 34.3 \\
\hline Finlanda & 45.6 & 45.0 & 48.3 & 47.4 & 48.8 & 49.6 & 51.8 & 49.4 & 51.1 & 52.4 \\
\hline Polonia & 53.3 & 53.3 & 54.2 & 53.1 & 52.9 & 54.6 & 55.6 & 55.4 & 54.6 & 47.8 \\
\hline $\begin{array}{l}\text { República } \\
\text { Checa }\end{array}$ & 38.8 & 41.6 & 42.0 & 42.7 & 43.4 & 45.0 & 43.9 & 42.7 & 43.2 & 42.8 \\
\hline Hungría & 77.9 & 76.7 & 75.5 & 75.3 & 76.2 & 79.3 & 80.3 & 79.3 & 77.9 & 84.6 \\
\hline Eslovenia & 45.4 & 45.6 & 46.8 & 47.0 & 52.7 & 53.1 & 61.0 & 61.0 & 70.2 & 77.1 \\
\hline Eslovaquia & 43.2 & 46.3 & 50.3 & 51.3 & 52.4 & 55.3 & 58.2 & 57.2 & 55.3 & 58.3 \\
\hline Estonia & 3.1 & 3.7 & 4.4 & 6.6 & 7.1 & 7.0 & 7.1 & 7.1 & 7.1 & 7.0 \\
\hline Lituana & 35.1 & 39.3 & 37.2 & 37.3 & 37.4 & 37.7 & 37.3 & 36.6 & 36.5 & 37.5 \\
\hline Malta & 68.8 & 72.0 & 74.0 & 71.7 & 70.7 & 74.3 & 75.0 & 75.7 & 72.6 & 75.2 \\
\hline Chipre & 113.2 & 117.0 & 126.6 & 127.2 & 130.0 & 131.8 & 143.9 & 155.4 & 158.5 & 160.0 \\
\hline Bulgaria & 15.7 & 16.2 & 15.9 & 18.1 & 18.0 & 17.6 & 17.7 & 17.1 & 18.6 & 19.9 \\
\hline Rumania & 33.7 & 37.3 & 36.1 & 35.7 & 36.6 & 38.0 & 38.0 & 38.4 & 37.3 & 38.4 \\
\hline Croacia & 50.6 & 52.7 & 53.3 & 53.7 & 54.9 & 56.8 & 59.0 & 60.9 & 65.8 & 66.5 \\
\hline
\end{tabular}

Fuente: Eurostat; $n d=$ no disponible.

Con respecto al nivel de endeudamiento medido en términos del PIB para Grecia y Portugal en los años de 2011 a 2014, muestran (cuadro 2) niveles superiores al 100 por ciento con respecto al PIB, mientras España para esos años tiene un promedio de endeudamiento con respecto al PIB de alrededor del 70 por ciento.
Estos hechos indican que los países de la Unión se mantenían dentro de los "límites" de un endeudamiento "manejable", implicando un alto riesgo frente a circunstancias externas (choques externos) como una crisis que dificulte el control gubernamental sobre tales 


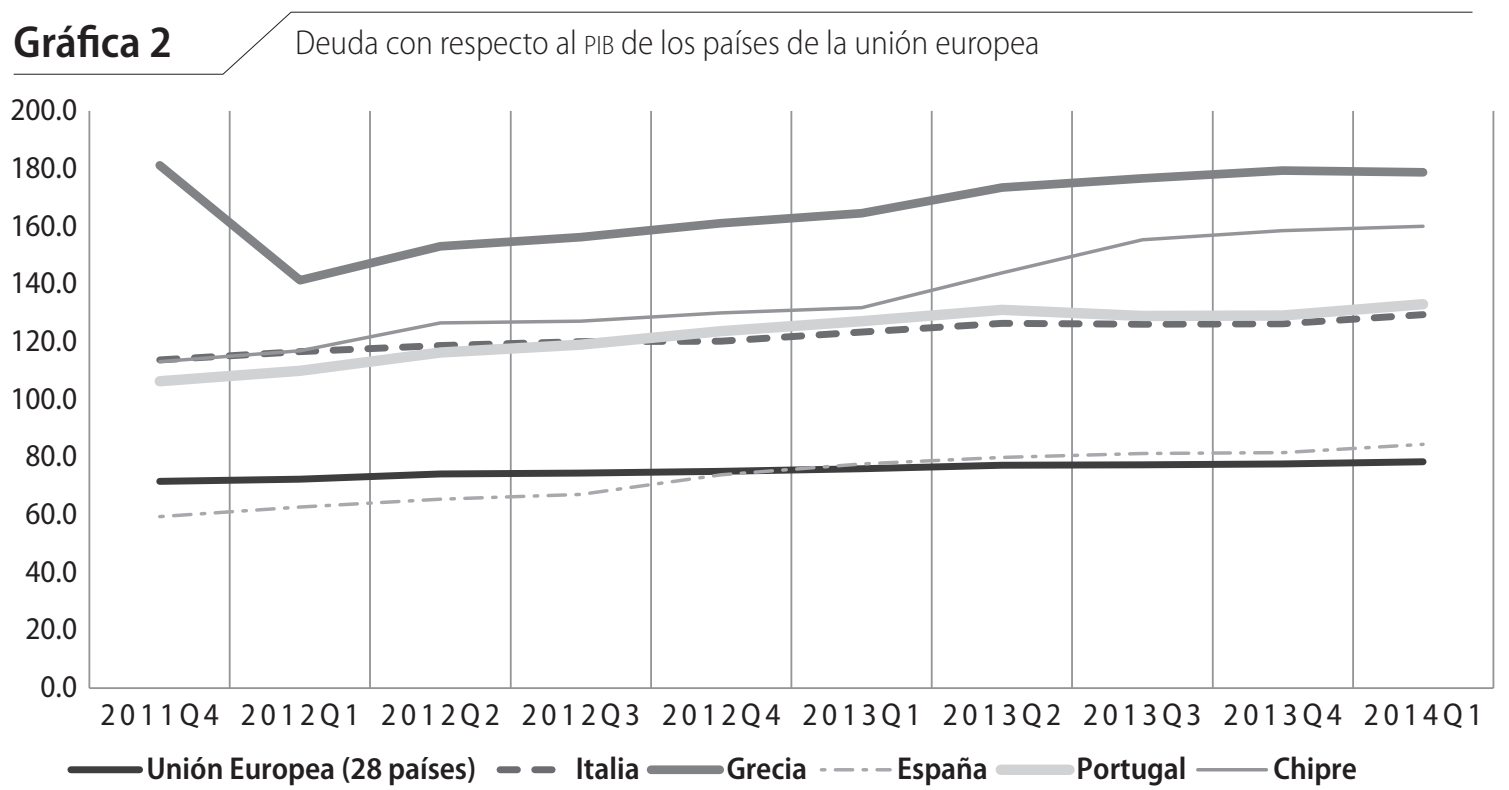

Fuente: elaboración propia con datos de Eurostat.

variables, -como en realidad sucedió- y en condiciones recesivas estos países tuvieron poco margen de maniobra de su política económica.

"El aumento de la deuda privada a cargo del sector privado es reciente: hubo un primer momento importante después de la entrada de Grecia en la zona euro, 2001; una segunda explosión de la deuda se inició a partir de 2007, cuando la ayuda financiera concedida a los bancos por la Reserva Federal de Estados Unidos por los gobiernos europeos y por el Banco Central Europeo (вСE), fue en parte reciclada por los banqueros hacia Grecia [...] En cambio el crecimiento de la deuda pública es más antiguo. Después de la deuda heredada de la dictadura de los coroneles, el recurso del préstamo sirvió desde los años noventa para rellenar el agujero producido en las finanzas públicas [...] endeudamiento en que el cayó gobierno griego para financiar la organización de los juegos olímpicos de 2004 en Atenas. "9

9 Damien Miller y E. Toussaint (2011). La deuda o la vida, editorial Icaria, p. 95
Frente a esta situación de sobreendeudamiento y ante la incapacidad de generar los ingresos suficientes para el pago de la deuda pública, las medidas de impulso de la demanda agregada recayeron exclusivamente en el área de las finanzas públicas, dado que la política monetaria, a través de los instrumentos del tipo de cambio y de la tasa de interés son variables manejadas por el Banco Central Europeo, no pueden ser utilizadas por los países integrantes de la Unión Europea, para hacer sus ajustes y corregir sus desequilibrios en el sector financiero interno y externo.

La crisis de la deuda se instaló en Europa, y arrastró dramáticamente a los actores europeos casi al borde de la salida de Grecia de la zona euro, al someter a este país a un riguroso recorte del gasto público en sectores sociales sensibles para la población griega, pero esto mismo se hizo extensivo a los países que alcanzaron altos niveles de endeudamiento. ${ }^{10}$

10 Para una revisión de los niveles de endeudamiento alcanzado por países europeos como Grecia Véase Toussaint (2011). 


\section{Santiaga Ánima Puentes | La Unión Europea y su problemática de integración}

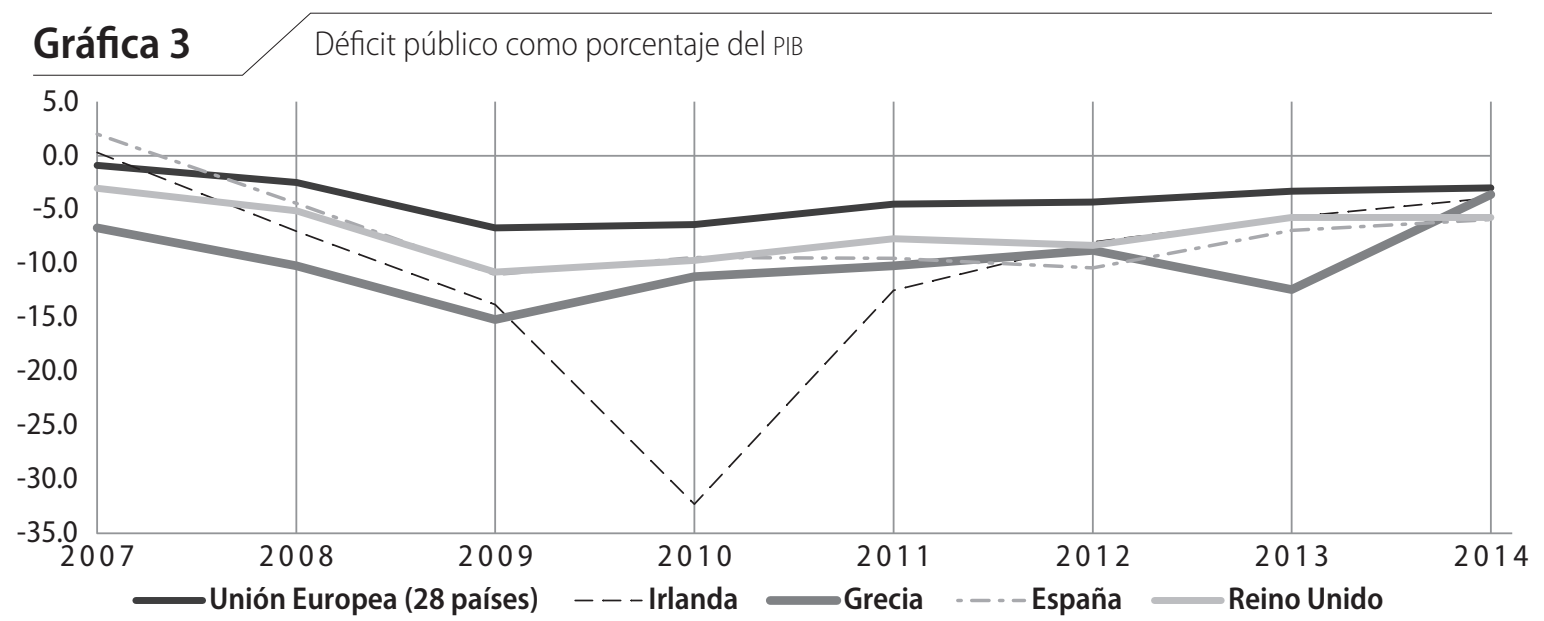

Fuente: Elaboración propia con datos de Eurostat.

Los problemas que enfrento Grecia ante los altos niveles de endeudamiento tuvieron como antecedente el crónico nivel de deuda que Grecia tenía previo a los juegos olímpicos, el problema se agudizo a partir de los mismos juegos. Además, llama la atención como pese a este problema estructural de endeudamiento griego, fue el entonces banco de inversión Goldman Sachs quién solapo la fragilidad financiera de Grecia al dar su aval para la entrada a la zona euro, ello a través, del uso de ingeniería financiera que oculto las verdaderas cifras de endeudamiento de dicho país.

Por otra parte, el problema de Chipre se debió a un colapso de su sistema bancario "altamente internacionalizado" que obligó al cierre de sus instituciones financieras y a que el gobierno asumiera los costos del saneamiento de la quiebra bancaria a cargo de las finanzas públicas, en consecuencia, Chipre tuvo que aceptar duras condiciones para tener acceso a los recursos con los cuales solventar su nivel de endeudamiento y corregir su déficit público.

Entre los países que tienen endeudamiento por debajo de lo establecido en Maastricht se encuentran Alemania, Luxemburgo, Suecia, Republica Checa, Dinamarca, Estonia, Lituania, Polonia, Eslovaquia y Finlandia, sin em- bargo, el promedio de endeudamiento de los 28 países es superior a lo acordado en el Tratado de integración de la Unión, situación que muestra la fragilidad en la que pueden caer varios países por los niveles tan altos de endeudamiento con respecto al PIB. Cabe notar que salvo Alemania el resto de los países con finanzas sanas tienen poco peso relativo en términos de su participación en la actividad económica.

\section{Aumento del déficit público}

El déficit gubernamental al estar asociado con la capacidad de equilibrar las fuentes de los ingresos con el destino de los mismos hacia actividades económicas y sociales, ha establecido que para no permitir que se gaste más de lo que ingresa en las arcas públicas, el Tratado de Maastricht le puso un límite numérico al déficit público no superior al 3 por ciento.

El comportamiento del balance público de las 28 economías de la UE se incrementó en más del doble a partir de 2009 cuando registró un -6.7 por ciento, mismo que se redujo a -4.5 por ciento en 2011 y para el año de 2014 se situó en lo establecido por las reglas de la UE en -3.0 por ciento. (Cuadro 3). 
Los países que vieron un aumento significativo del déficit en 2009 fueron, Grecia -15.2, Irlanda -13.8, España -11.0 y el Reino Unido en 10.8 por ciento, aunque este último país no ha adoptado el Euro, conserva el manejo de su política monetaria por ende puede realizar los ajustes de sus balances internos y externos tanto en su política fiscal como cambiaria y comercial. (Gráfica 3). Para el año 2010 Irlan$\mathrm{da}^{11}$ fue el país que registró el más alto déficit público y el país que ajusto su gasto público para reducir drásticamente el déficit y mitigar el alto nivel de endeudamiento.

Respecto del comportamiento del déficit público como porcentaje del PIB (Cuadro 3) podemos constatar que de 2007 a 2014 la mayoría de los 28 países que integran la ue han incurrido en un déficit superior al 3 por ciento. Este comportamiento del déficit nos obliga a preguntarnos ¿hasta qué grado es realista mantener

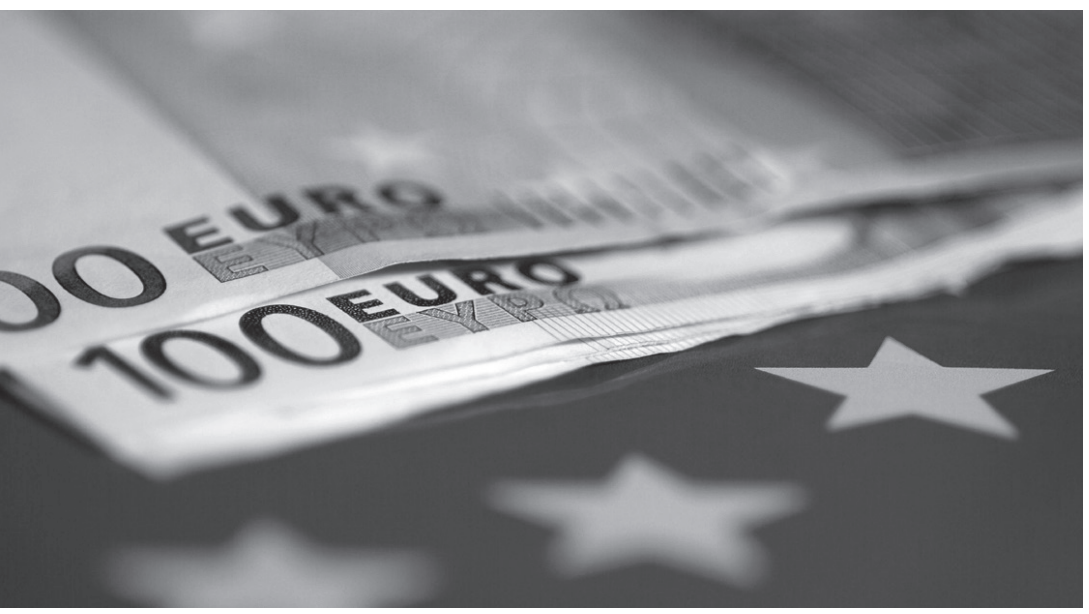

11 Irlanda registró en ese año un espectacular déficit de -32.3 como puede observarse en la gráfica 3. Este hecho se debió a circunstancias similares a las de México al asumir el Estado la deuda privada como pública a través del conocido Fobaproa-IPAB. En el caso de Irlanda el Parlamento aceptó la conversión de la deuda privada de los cuatro bancos más importantes en deuda pública, y el gobierno irlandés asumió esa deuda en condiciones en que sus finanzas públicas estaban en una situación prácticamente de insolvencia y fragilidad fiscal. una situación restrictiva en el equilibrio de las finanzas públicas?, si en situación de crisis económica existe la necesidad de aumentar el gasto para mitigar los efectos recesivos de la crisis.

La Unión Europea dada la evidencia de los resultados de las políticas de ajuste, (de aumento del desempleo, caídas en la producción y deterioro de las condiciones sociales de las poblaciones más vulnerables de la Unión), estaría en posibilidad de reconsiderar y reevaluar las políticas hasta ahora aplicadas, es decir ¿Qué es más importante para la UE mantener la zona euro o preservar las condiciones de vida de los europeos?

El reto que sigue planteando la viabilidad del proceso de integración europea es continuar por la ruta o ajustarse a los cambios dinámicos de los mercados comerciales y financieros internacionales y entonces permitirse establecer nuevos acuerdos que permitan cierto grado de flexibilidad de las políticas monetarias, cambiarias y fiscales que estén a tono con la resolución de problemas sociales a tendiendo el lacerante problema del empleo y la migración ajustando las medidas a las circunstancias económicas globales que la misma realidad muestra.

La Unión Europea ha logrado al observar las cifras presentadas si resintió los efectos de la crisis global, sin embargo, logró reaccionar aumentando los niveles de demanda agregada con un incremento del gasto público, pero esto fue temporal no se resuelve de fondo la vulnerabilidad y fragilidad de la integración misma que se ve sometida a pruebas cada vez más fuertes por también más fuertes crisis globales.

Los países que comandan el proceso de integración como son Alemania y Francia también, a consecuencia de la crisis financiera, aumentaron su déficit público entre 2009-2010 para después a partir de 2011 disminuirlo con programas de ajuste. Es decir, estos países pa- 
Santiaga Ánima Puentes | La Unión Europea y su problemática de integración

Cuadro 3

Déficit público como porcentaje del PIB

\begin{tabular}{|c|c|c|c|c|c|c|c|c|}
\hline País/Año & 2007 & 2008 & 2009 & 2010 & 2011 & 2012 & 2013 & 2014 \\
\hline uE (28 países) & -0.9 & -2.5 & -6.7 & -6.4 & -4.5 & -4.3 & -3.3 & -3.0 \\
\hline Bélgica & 0.1 & -1.1 & -5.4 & -4.0 & -4.1 & -4.1 & -2.9 & -3.1 \\
\hline Luxemburgo & 4.2 & 3.3 & -0.5 & -0.5 & 0.5 & 0.2 & 0.7 & 1.4 \\
\hline Holanda & 0.2 & 0.2 & -5.4 & -5.0 & -4.3 & -3.9 & -2.4 & -2.4 \\
\hline Alemania & 0.2 & -0.2 & -3.2 & -4.2 & -1.0 & -0.1 & -0.1 & 0.3 \\
\hline Francia & -2.5 & -3.2 & -7.2 & -6.8 & -5.1 & -4.8 & -4.1 & -3.9 \\
\hline Italia & -1.5 & -2.7 & -5.3 & -4.2 & -3.5 & -3.0 & -2.9 & -3.0 \\
\hline Reino Unido & -3.0 & -5.1 & -10.8 & -9.7 & -7.7 & -8.3 & -5.7 & -5.7 \\
\hline Irlanda & 0.3 & -7.0 & -13.8 & -32.3 & -12.5 & -8.0 & -5.7 & -3.9 \\
\hline Dinamarca & 5.0 & 3.2 & -2.8 & -2.7 & -2.1 & -3.6 & -1.3 & -1.5 \\
\hline Grecia & -6.7 & -10.2 & -15.2 & -11.2 & -10.2 & -8.8 & -12.4 & -3.6 \\
\hline España & 2.0 & -4.4 & -11.0 & -9.4 & -9.5 & -10.4 & -6.9 & -5.9 \\
\hline Portugal & -3.0 & -3.8 & -9.8 & -11.2 & -7.4 & -5.7 & -4.8 & -7.2 \\
\hline Austria & -1.3 & -1.4 & -5.3 & -4.4 & -2.6 & -2.2 & -1.3 & -2.7 \\
\hline Suecia & 3.3 & 2.0 & -0.7 & 0.0 & -0.1 & -0.9 & -1.4 & -1.7 \\
\hline Finlanda & 5.1 & 4.2 & -2.5 & -2.6 & -1.0 & -2.1 & -2.5 & -3.3 \\
\hline Polonia & -1.9 & -3.6 & -7.3 & -7.5 & -4.9 & -3.7 & -4.0 & -3.3 \\
\hline República Checa & -0.7 & -2.1 & -5.5 & -4.4 & -2.7 & -4.0 & -1.3 & -1.9 \\
\hline Hungría & -5.1 & -3.6 & -4.6 & -4.5 & -5.5 & -2.3 & -2.5 & -2.5 \\
\hline Eslovenia & -0.1 & -1.4 & -5.9 & -5.6 & -6.6 & -4.1 & -15.0 & -5.0 \\
\hline Eslovaquia & -1.9 & -2.3 & -7.9 & -7.5 & -4.1 & -4.2 & -2.6 & -22.8 \\
\hline Estonia & 2.7 & -2.7 & -2.2 & 0.2 & 1.2 & -0.3 & -0.1 & 0.7 \\
\hline Letonia & -0.7 & -4.1 & -9.1 & -8.5 & -3.4 & -0.8 & -0.9 & -1.5 \\
\hline Lituana & -0.8 & -3.1 & -9.1 & -6.9 & -8.9 & -3.1 & -2.6 & -0.7 \\
\hline Malta & -2.3 & -4.2 & -3.3 & -3.2 & -2.6 & -3.6 & -2.6 & -2.1 \\
\hline Chipre & 3.2 & 0.9 & -5.5 & -4.8 & -5.7 & -5.8 & -4.9 & -8.9 \\
\hline Bulgaria & 1.1 & 1.6 & -4.1 & -3.2 & -2.0 & -0.6 & -0.8 & -5.8 \\
\hline Rumania & -2.9 & -5.6 & -9.1 & -6.9 & -5.4 & -3.2 & -2.2 & -1.4 \\
\hline Croacia & -2.4 & -2.7 & -5.8 & -5.9 & -7.8 & -5.3 & -5.4 & -5.6 \\
\hline
\end{tabular}

Fuente: Eurostat.

decieron problemas de déficit público, mismos que de inmediato fueron corregidos debido a su poder económico y soporte de las demás economías, sabiendo que con su intervención establecían medidas de ajuste a los países con mayores problemas de endeudamiento, ya que en caso de no hacerlo los mercados financieros los habrían castigado dificultando todavía más una solución integral que preserve el proceso de integración.

De los años de 2011 a 2014 el déficit público fue una constante para la Unión Europea, no obstante, el comportamiento del déficit no fue uniforme ni estuvo acorde a lo establecido en el Tratado de Maastricht. Aunque algunos países si cumplieron con la regla la mayor par- 
te no lo hizo, implicando que algunos de ellos recurrieron a políticas de incrementos del gasto público para evitar los efectos recesivos en sus economías. Alemania y Francia, además tuvieron que asumirse como fiadores del resto de las economías frente a sus acreedores combinando el uso de sus ahorros con medidas de ajuste económico.

La política económica de la UE en un contexto de recesión económica obliga a tomar medidas de carácter keynesiano, es decir de expansión de la demanda agregada vía el componente autónomo del gasto público, con la finalidad de tratar de contrarrestar la caída en la actividad económica.

El mercado financiero para acceder a los recursos se vio restringido por lo extendido de los instrumentos financieros conocidos como bonos tóxicos, que al derrumbarse la confianza sobre los mismos y su incapacidad para solventar los pagos correspondientes por concepto de intereses, ocasionaron en el mercado financiero una astringencia de crédito que frenó las expectativas de inversión ya no solo en el sector financiero sino también en el sector real de la actividad económica de los países que resintieron los efectos de la crisis subprime ${ }^{12}$ en Estados Unidos. Igual ocurrió con los países de la Unión Europea que también resintieron los efectos de la globalización financiera.

12 Se denomina crisis de subprime a los bonos colocados en los mercados financieros que eran respaldados por las inversiones en el sector inmobiliario norteamericano, sin más respaldo que el pago de los insolventes dueños de viviendas, que al dejar de pagar mostraron la fragilidad de las hipotecas de las viviendas, ocasionando con ello la pérdida de valor de los instrumentos financieros colocados en los principales mercado financieros internacionales que frente a la insolvencia su pérdida de valor ocasiono el colapso de los mercados que comerciaban con dichos instrumentos.

\section{Gasto Público y Desempleo en los países de la Unión}

Como se había mencionado, el impacto de la crisis en 2009 obligó a los gobiernos de la Unión Europea a incrementar sus niveles de gasto público, buscando paliar los efectos recesivos que provenían del sector financiero y golpeaban a la actividad productiva, es decir se buscó reactivar de lado de la demanda agregada el componente autónomo del gasto público y contrarrestar los efectos recesivos en la demanda agregada y el empleo de la crisis global en los países de la Unión.

La medida de expansión gubernamental, como se ha señalado, fue que todos los países incrementaron su gasto público en 2009 siendo los más altos Francia, Dinamarca y Finlandia, con ello en el corto plazo incentivar una política fiscal anticiclíca que no permita caer en una profunda recesión; a pesar de los efectos positivos de dicha medida, para 2010, el gasto se redujo en todos los países excepto en Irlanda donde se incrementó al 65.5 como porcentaje del PIB.

Así, prácticamente todos los países de la UE (cuadro 4) aumentaron sus niveles del gasto público como porcentaje del PIB, para posteriormente estabilizarse en un promedio de 50 por ciento; entre los países que incrementaron el gasto publico arriba del 55 por ciento del PIB en 2010 fueron Irlanda, Dinamarca, Francia y Finlandia.

Debido al manejo que tienen los gobiernos en las variables fiscales y de gasto público, ante la falta de manejo de la política monetaria, se aplicó una política contra cíclica convencional para ajustar el desequilibrio vía el gasto, en un primer momento, en especial durante 2009 ante la gravedad de la crisis se recurrió al aumento del gasto; en un segundo momento, a partir de 2010 se aplicaron medidas de 
restricción y reducción de recursos públicos para equilibrar las finanzas públicas en el largo plazo, a costa de afectar a los sectores sociales que se veían beneficiados con el apoyo del sector público. Es por eso, que en los países que requerían recursos para salir de la crisis tenían por obligación que realizar recortes a sus programas particularmente sociales con la finalidad de evitar un mayor endeudamiento y déficit público.

Cuadro 4

Déficit público como porcentaje del PIB

\begin{tabular}{|c|c|c|c|c|c|c|c|}
\hline País/Año & 2007 & 2008 & 2009 & 2010 & 2011 & 2012 & 2013 \\
\hline UE (28 países) & 45.5 & 47.0 & 51.0 & 50.6 & 49.0 & 49.4 & 49.1 \\
\hline Bélgica & 48.2 & 49.8 & 53.7 & 52.6 & 53.6 & 55.1 & 54.5 \\
\hline Luxemburgo & 36.3 & 39.1 & 45.2 & 43.5 & 42.6 & 43.9 & 43.5 \\
\hline Holanda & 45.3 & 46.2 & 51.4 & 51.3 & 49.9 & 50.4 & 49.8 \\
\hline Alemania & 43.5 & 44.1 & 48.3 & 47.9 & 45.2 & 44.7 & 44.7 \\
\hline Francia & 52.6 & 53.3 & 56.7 & 56.6 & 55.9 & 56.7 & 57.1 \\
\hline Italia & 47.6 & 48.6 & 51.9 & 50.5 & 49.7 & 50.6 & 50.6 \\
\hline Reino Unido & 43.3 & 47.1 & 50.8 & 49.9 & 47.9 & 48.1 & 47.1 \\
\hline Irlanda & 36.7 & 42.8 & 48.2 & 65.5 & 47.1 & 42.6 & 42.9 \\
\hline Dinamarca & 50.8 & 51.5 & 58.1 & 57.7 & 57.7 & 59.4 & 57.2 \\
\hline Grecia & 47.5 & 50.6 & 54.0 & 51.4 & 51.9 & 53.4 & 58.5 \\
\hline España & 39.2 & 41.1 & 46.2 & 46.3 & 45.7 & 47.8 & 44.8 \\
\hline Portugal & 44.4 & 44.8 & 49.8 & 51.5 & 49.3 & 47.4 & 48.7 \\
\hline Austria & 48.6 & 49.3 & 52.6 & 52.8 & 50.8 & 51.6 & 51.3 \\
\hline Suecia & 51.0 & 51.7 & 54.9 & 52.3 & 51.5 & 52.0 & 52.8 \\
\hline Finlanda & 47.4 & 49.2 & 56.1 & 55.8 & 55.1 & 56.7 & 58.5 \\
\hline Polonia & 42.2 & 43.2 & 44.6 & 45.4 & 43.4 & 42.2 & 41.9 \\
\hline República Checa & 41.0 & 41.1 & 44.7 & 43.7 & 43.2 & 44.5 & 42.3 \\
\hline Hungría & 50.7 & 49.2 & 51.4 & 50.0 & 50.0 & 48.7 & 50.0 \\
\hline Eslovenia & 42.3 & 44.1 & 48.7 & 49.5 & 49.9 & 48.4 & 59.4 \\
\hline Eslovaquia & 34.2 & 34.9 & 41.6 & 39.8 & 38.9 & 38.2 & 38.7 \\
\hline Estonia & 34.0 & 39.7 & 44.7 & 40.5 & 37.6 & 39.5 & 38.3 \\
\hline Letonia & 36.0 & 39.1 & 43.6 & 43.4 & 38.4 & 36.5 & 36.1 \\
\hline Lituana & 35.3 & 37.9 & 44.9 & 42.3 & 38.7 & 36.1 & 34.4 \\
\hline Malta & 41.8 & 43.3 & 42.5 & 41.2 & 41.4 & 43.1 & 43.7 \\
\hline Chipre & 41.3 & 42.1 & 46.2 & 46.2 & 46.3 & 45.8 & 45.8 \\
\hline Bulgaria & 39.2 & 38.4 & 41.4 & 37.4 & 35.6 & 35.8 & 38.7 \\
\hline Rumania & 38.2 & 39.3 & 41.1 & 40.1 & 39.4 & 36.7 & 35.0 \\
\hline Croacia & 44.0 & 43.4 & 46.2 & 46.9 & 48.1 & 46.0 & 46.1 \\
\hline
\end{tabular}

En este contexto la llamada Troika (integrada por El Consejo Europeo, Fondo Monetario Internacional (FMI) y el Banco Central Europeo $\mathrm{BCE}$ ) ante la astringencia de liquidez internacional para otorgar créditos condicionaron el acceso a los mismos solo si se cumplían duros programas de ajustes en el gasto público que afectaban al sector social, el caso que más repercusiones internacionales tuvo fue el de Grecia.

Fuente: Eurostat. 
Cuadro 5

Tasa de desempleo

\begin{tabular}{|c|c|c|c|c|c|}
\hline País/año & $2013 \mathrm{a} /$ & $2014 \mathrm{~b} /$ & País/año & $2013 \mathrm{a} /$ & $2014 \mathrm{a} /$ \\
\hline EU (28 países) & 10.6 & 10.2 & Finlandia & 8.3 & 8.7 \\
\hline Bélgica & 8.5 & 8.5 & Polonia & 10 & 9 \\
\hline Luxemburgo & 6.2 & 6.3 & República Checa & 6.7 & 6 \\
\hline Holanda & 7 & 6.7 & Hungría & 8.7 & nd \\
\hline Alemania & 5.2 & 4.9 & Eslovenia & 9.9 & 9.8 \\
\hline Francia & 10.2 & 10.3 & Eslovaquia & 14 & 13.8 \\
\hline Italia & 12.4 & 12.6 & Estonia & 8.3 & nd \\
\hline Reino Unido & 7 & nd & Letonia & 11.5 & nd \\
\hline Irlanda & 12 & 11.5 & Lituania & 11.3 & 11.5 \\
\hline Dinamarca & 7.1 & 6.6 & Malta & 6.4 & 5.7 \\
\hline Grecia & 27.4 & nd & Chipre & 16.5 & 14.9 \\
\hline España & 25.6 & 24.5 & Bulgaria & 12.8 & 11.5 \\
\hline Portugal & 15.2 & 14 & Rumania & 7.3 & 7 \\
\hline Austria & 5.1 & 4.9 & Croacia & 17.1 & 16.2 \\
\hline Suecia & 8 & 7.8 & & & \\
\hline
\end{tabular}

Fuente: Eurostat.

a/ mes de diciembre; b/mes de julio; $n d=$ no disponible.

La situación de crisis se expresó en el aumento del desempleo como consecuencia de la caída de la inversión y la falta de liquidez en los mercados internacionales. Países como España ${ }^{13}$ resintieron los problemas del creciente desempleo, situación que hoy día sigue sin resolverse en dicho país.

Los efectos recesivos de las economías de la Unión Europea se manifestaron de diferentes maneras, pero quienes más lo resintieron, fueron los países con altos niveles de endeudamiento y frágil integración a la Unión, así las tasas de desempleo más altas en los últimos años las registraron en países como Grecia y España con un 27 y 25 por ciento respectivamente para 2013. (Cuadro 5). Y los países con

13 Hay que hacer notar que el impacto del desempleo es diferenciado tanto en términos de edad como de distribución regional, castigando a los núcleos más jóvenes de la población y a las regiones autónomas más pobres como Extremadura. mayor fortaleza económica tuvieron menores tasas de desempleo como fueron Alemania y Austria con tasas similares de 5.2 (5.1) y 4.9 para los años de 2013 y 2014.

\section{Conclusión}

En conjunto se observa que hoy más que nunca los países integrantes de la Unión Europea son más vulnerables y frágiles frente a choques externos de los mercados financieros internacionales. En ese sentido, el proceso de integración económica de la Unión Europea no se ha traducido en una fortaleza que resista los movimientos especulativos y cíclicos de los mercados financieros, como era el objetivo original.

En este contexto, el país que está asumiendo el liderazgo de la Unión, Alemania por ser económicamente más importante de la Unión en términos de su aportación al PIB de la región, ha establecido medidas que buscan pre- 
servar el proceso de integración económica, incluso promoviendo medidas de política fiscal anticiclíca con fuerte incremento del gasto público en clara violación a el Tratado de Maastricht, sin embargo a pesar de ser el país que asume el costo de los apoyos al resto de los integrantes de la Unión, enfrenta serios problemas de endeudamiento, déficit público y creciente desempleo; aunque no a niveles de Grecia, España, Portugal e Italia.

La crisis de la deuda soberana ha llevado a poner en cuestión el proceso de integración plena de la Unión, llegándose a mencionar la posible salida de Grecia de la Zona Euro, situación que pondría en entredicho la viabilidad de la propia integración económica de la región.

La salida de Grecia implicaría, en términos de los procesos de integración europea, un retroceso de consecuencias no solo económicas sino de carácter social y político, tales como: la pérdida de confianza en la unidad europea, presiones sobre el tipo de cambio y la viabilidad del euro, acentuando la restricción de la liquidez internacional y afectando el comercio en la zona euro y en el mundo. Y aunque el presente trabajo no aborda específicamente la situación de inestabilidad de la integración económica de la zona a raíz del llamado crisis del Brexit, por la no continuación de Inglaterra en el proceso de integración, se reconoce que la Unión Europea enfrenta el reto de continuar o fragmentarse.

El proceso de integración de la UE se ve enfrentado a problemáticas que como identifica- mos se encuentran en las reacciones de política anticiclícas, que implementaron los países de la Unión, para aminorar los impactos devastadores de la crisis financiera global, aunado a esto la problemática se agudiza con los conflictos en Ucrania-Crimea-Rusia y la migración masiva de los refugiados sirios, ambos fenómenos de reciente arribo.

La situación del proceso de integración económico europeo está supeditado a la buena marcha de la actividad económica y del impulso a los agregados macroeconómicos fundamentales que permitan el crecimiento económico de la región, pero si siguen enfrentando fluctuaciones en su actividad económica que no logran contrarrestar con su proceso de integración los resultados serán un incremento de países con fuertes problemas económicos, como ya se destacó en el trabajo, a raíz de la crisis global de 2009.

Los países integrantes de la Unión en su conjunto, ante la severa caída de sus niveles de crecimiento, se han visto obligados a responder con políticas expansivas en la demanda agregada (vía aumento del déficit público y de los niveles de endeudamiento), en consecuencia, prácticamente la totalidad de los países de la zona euro no han cumplido, como lo señalamos, con lo establecido en Maastricht durante el periodo 2007-2014.

Ha sido el desempleo la expresión más dura y difícil de la situación crítica que le ha tocado vivir a la UE, amenazando incluso su propia existencia; sin embargo, los países de la 
zona siguen permaneciendo en ella a pesar de la crisis que han padecido, aun considerando lo acontecido con el Brexit, la mayoría de los países y de sus poblaciones siguen considerando continuar con el proceso de integración y verían más grave salir de la Unión que permanecer en la misma.

La situación en la que ha entrado la Unión Europea la hacen un protagonista fundamental y que no debe de concentrarse únicamente en sus procesos internos, sino que su peso específico adquirido en el ámbito económico y político internacional, la obligan a actuar de manera más abierta y responsable en el ámbito global. Europa se encuentra bajo nuevas circunstancias, el reto inmediato que tiene es resolver sus problemas internos, para posteriormente actuar como un verdadero bloque frente a problemas ajenos a sus países miembros.

Si se sigue dificultando la resolución de sus problemas inmediatos, veremos acrecentarse los conflictos, mismos que como se vio recientemente con el Brexit, complican el escenario actual de integración económica y retardan, sino es que cuestionan todo el proceso mismo de integración europea.

\section{Bibliografía}

BARry Eichengreen, (2007). The European economic since 1945, Princeton University Press

Alicia Girón y Marcia Solorza, (2013). Euro$p a$, deudas soberanas y financiación, UNAM.

Jean Pisani-Ferry, (2012). El despertar de los demonios. La crisis del euro y como salir de ella, Antoni Bosch, Barcelona.

Francisco Rodríguez Ortiz, (2012). Las máscaras de la crisis. Europa a la deriva, editorial Catarata, Madrid.

(2004). Europa:

entre la integración monetaria y la crisis económica mundial, editorial Catarata, Madrid.

Ramón Tamames, y Begoña Huerta, (2010). Estructura Económica Internacional, Alianza Editorial, Madrid.

ERIC Toussaint, et al. (2011). La deuda o la vida. Europa en el ojo del huracán. Icaria, Barcelona. 